\title{
Anonymity preserving and Lightweight Multi-Medical Server Authentication Protocol for Telecare Medical Information System
}

\author{
Ruhul Amin, SK Hafizul Islam, Senior Member, IEEE, Prosanta Gope, Kim-Kwang Raymond Choo, Senior \\ Member, IEEE, and Nachiket Tapas
} \begin{abstract}
reuse of any copyrighted component of this work in other works.
Abstract-Electronic health systems, such as Telecare Medical Information System (TMIS), allow patients to exchange their health information with a medical center/doctor for diagnosis in real-time, and across borders. Given the sensitive nature of health information/medical data, ensuring the security of such systems is crucial. In this paper, we revisit Das et al.'s authentication protocol, which is designed to ensure patient anonymity and untraceability. Then, we demonstrate that the security claims are invalid, by showing how both security features can be compromised. We also demonstrate that the protocol suffers from new smartcard launch attacks. To mitigate such design flaws, we propose a new lightweight authentication protocol using the cryptographic hash function for TMIS. We then analyze the security of the proposed protocol using AVISPA and Scyther, two widely used formal specification tools. The performance analysis demonstrates that our protocol is more efficient than other competing protocols.
\end{abstract}

(c) 2018 IEEE. Personal use of this material is permitted. Permission from IEEE must be obtained for all other uses, in any current or future media, including reprinting/republishing this material for advertising or promotional purposes, creating new collective works, for resale or redistribution to servers or lists, or

Index Terms-AVISPA; Multi-Medical system; Authentication; Anonymity; Telecare Medical Information System; Scyther.

\section{INTRODUCTION}

A $\mathrm{N}$ efficient electronic health system can facilitate or inform medical decision-making based on accurate and up-to-date information, such as the most up-to-date health / medical condition of the patient. It can be challenging to make a medical diagnosis for a new patient since the medical practitioner does not access to the patient's prior medical history and other relevant information in real-time, since this is the first time that this medical practitioner is seeing the patient. This limitation can be mitigated in an electronic health system, since the medical practitioner from a participating healthcare institution can obtain real-time access to the patient's medical history and other relevant information stored in the server of another healthcare institution. Clearly,

R. Amin is with the Department of Computer Science and Engineering, Dr. Shyama Prasad Mukherjee International Institute of Information Technology, Naya Raipur, Chhattisgarh 493661, India. E-mail: amin_ruhul@live.com

S.H. Islam is with the Department of Computer Science and Engineering, Indian Institute of Information Technology Kalyani, West Bengal 741235, India. Email:hafi786@gmail.com

P. Gope is with the School of Engineering and Computer Science, University of Hull Hull, HU6 7RX, United Kingdom. E-mail: prosanta@comp.nus.edu.sg

K-K. R. Choo is with the Department of Information Systems and Cyber Security and the Department of Electrical and Computer Engineering, The University of Texas at San Antonio, San Antonio, TX 78249, USA. E-mail: raymond.choo@fulbrightmail.org

N. Tapas is with the Department of Engineering, University of Messina, Italy 98158. E-mail: ntapas@unime.it ensuring data confidentiality, integrity, and privacy is crucial in such systems. However, requirements may vary between different electronic health systems, for example due to the privacy legislation in the jurisdiction where the systems are hosted. Examples of electronic health systems include the following [1]:

- Electronic Health Record (ERH) system is designed to maintain patient records, and facilitate e-prescribing, clinical administration, e-booking and digital imaging, and archiving systems.

- Telemedicine and telehealth systems allow the dissemination and storage of health and other medical data over the Internet to facilitate medical diagnosis and other medical services; thus, improving patient access and optimizing use of digital health assets.

- Clinical health IT systems include radiology, nursing systems, computer-assisted diagnostics, medical imaging, and surgery training and planning systems. Such systems facilitate physicians in providing more accurate diagnoses and treatments to patients.

- Online health IT systems include online health portals, which can be accessed by patients to obtain information about health related services (e.g. book an appointment and check on the reports of one's recent laboratory tests).

- Specialized electronic health systems include those used by researchers for public health data collection and analysis, such as bio-statistical programs for infectious diseases, drug development, and outcomes analysis.

In a Telecare Medical Information System (TMIS), patient anonymity is crucial and violation of patient privacy can have serious legal ramifications. Hence, cryptographic techniques are generally used to protect patient anonymity and data privacy [4], [5], [6], [3], [7], [8], [9], [2], [14], [17]. In 2015, for example, Amin and Biswas [17] proposed a conceptual architecture for the distributed medical system, where any registered patient can directly exchange medical data with participating medical practitioner(s). The authors also proposed a hash function based authentication protocol using smartcard. They claimed that their protocol is efficient and secure against several security threats. Das et al. [18], however, revealed a number of security weaknesses in Amin and Biswas's protocol [17] and presented an improved protocol. Independently, Lin et al. [19] also noted that the protocol of Amin and Biswas is insecure against off-line password guessing attack, 
insider attack and impersonation attack. In 2017, Liu et al. [20] also presented several security weaknesses of Das et al.'s [18] protocol as well as an improved protocol.

In the next section, we will revisit the protocol of Das et al. [18] before demonstrating in Section III that it is not capable of ensuring anonymity, as well as being insecure against traceability attack, and other smartcard related attacks. Hence, we propose an efficient authentication protocol that can be deployed in environment such as the TIMS architecture proposed by Amin and Biswas [17] - see Section IV. The security and performance analysis of our protocol are presented in Sections V and VI, respectively. We conclude the paper in Section VII.

\section{REVISITING DAS ET AL.'s PROTOCOL}

The protocol of Das et al. [18] consists of the following phases: (i) Medical-server registration phase, (ii) patient registration phase, (iii) login phase, (iv) authentication and session key agreement phase, (v) password and biometric update phase, and (vi) dynamic medical-server addition phase - see Sections II-A to II-D.

\section{A. Medical-server Registration Phase}

The medical-server $M S_{j}(1 \leq j \leq m)$ selects an identity $I D_{m j}$, and sends it to the central medical registration server $M R S$. Upon receiving the request, $M R S$ calculates a secret key $X_{m j}=h\left(I D_{m j} \| X_{c}\right)$ for $M S_{j}$, where $X_{c}$ denotes the secret key of $M R S$. Then, $M R S$ sends $X_{m j}$ to $M S_{j}$ securely. Note that $M S_{j}$ keeps $\left\langle I D_{m j}, X_{m j}\right\rangle$ in the database. For $m^{\prime}$ additional medical servers $M S_{j}\left(m^{\prime}+1 \leq j \leq m+m^{\prime}\right)$, $M R S$ selects an unique identity $I D_{a m j}$, calculates $X_{a m j}=$ $h\left(I D_{a m j} \| X_{c}\right)$, and stores $\left\langle I D_{a m j}, X_{c}\right\rangle$ in the memory for all additional medical servers $M S_{j}$. These information will be used ensure scalability, if needed.

\section{B. Patient Registration Phase}

In this phase, the following operations/steps are undertaken. Step 1. The patient $U_{p}$ enters $I D_{p}$ (identity), $P W_{p}$ (password), and $B_{p}$ (biometric) at the sensor device. $U_{p}$ creates a random number $K_{p}$ and computes $R P W_{p}=h\left(I D_{p}\left\|K_{p}\right\| P W_{p}\right)$. Further, $U_{p}$ utilizes the fuzzy extractor [27], [28] to calculate $\operatorname{Gen}\left(B_{p}\right)=\left(\sigma_{p}, \tau_{u}\right)$. Now, $U_{p}$ sends $\left\langle I D_{p}, R P W_{p}\right\rangle$ to $M R S$ via a secure network.

Step 2. On receiving $\left\langle I D_{p}, R P W_{p}\right\rangle, M R S$ calculates $A_{m j}=$ $h\left(I D_{p} \| X_{m j}\right) \oplus R P W_{p}, P_{m j}=h\left(I D_{m j} \| X_{m j}\right) \oplus R P W_{p}$ for all $\left(1 \leq j \leq m+m^{\prime}\right)$. Now, $M R S$ issues a new smartcard and writes $\left\langle I \bar{D}_{m j}, A_{m j}, P_{m j}, h(\cdot), \operatorname{Gen}(\cdot), \operatorname{Rep}(\cdot), t\right\rangle$ into the memory of the smartcard, where $1 \leq j \leq m+m^{\prime}$ and $t$ denotes error-tolerance threshold value for fuzzy extractor.

Step 3. On getting the smartcard, $U_{p}$ computes $E_{p}=h\left(I D_{p} \|\right.$ $\left.\sigma_{p}\right) \oplus K_{p}$ and $F_{p}=h\left(I D_{p}\left\|R P W_{p}\right\| \sigma_{p}\right)$. Finally, $U_{p}$ inserts $E_{p}$ and $F_{p}$ into the smartcard.

\section{Login Phase}

This phase is executed to login to any of the participating medical servers, including those belonging to other healthcare institutions. All operations performed in this phase are given below.

Step 1. $U_{p}$ inserts the smartcard into the smartcard-reader and enters $I D_{p}, P W_{p}$ and $B_{p}$. Now, the smartcard calculates $\sigma_{p}^{*}=\operatorname{Rep}\left(B_{p}, \tau_{p}\right)$ [27], [28], $K_{p}^{*}=h\left(I D_{p} \| \sigma_{p}^{*}\right) \oplus E_{p}$, $R P W_{p}^{*}=h\left(I D_{p}\left\|K_{p}^{*}\right\| P W_{p}\right), F_{p}^{*}=h\left(I D_{p}\left\|R P W_{p}^{*}\right\|\right.$ $\left.\sigma_{p}^{*}\right)$ and checks whether $F_{p}^{*}=? F_{p}$. If $F_{p}^{*} \neq F_{p}$, then the smartcard aborts the session, otherwise the inputted information is considered to be correct.

Step 2. The smartcard now creates a random number $R_{p}$ and timestamp $T S_{p}$, then computes $M G_{1}=A_{m j} \oplus R P W_{p}^{*}=$ $h\left(I D_{p} \| X_{m j}\right), M G_{2}=P_{m j} \oplus R P W_{p}^{*}=h\left(I D_{m j} \| X_{m j}\right)$, $M G_{3}=I D_{p} \oplus M G_{2}, M G_{4}=I D_{p} \oplus M G_{1} \oplus R_{p}$ and $M G_{5}=h\left(M G_{1}\left\|M G_{3}\right\| M G_{4}\left\|R_{p}\right\| T S_{p}\right)$. Now, the smartcard sends $\left\langle I D_{m j}, I D_{p k}, M G_{3}, M G_{4}, M G_{5}, T S_{p}\right\rangle$ as login message to $M S_{j}$ via any unreliable network.

\section{Authentication and Session Key Agreement Phase}

This phase authenticates the involved entities and negotiates a common session key for secure information exchange.

Step 1. On receiving the login message $\left\langle I D_{m j}, I D_{p k}, M G_{3}, M G_{4}, M G_{5}, T S_{p}\right\rangle$ at time $T S_{m j}$, $M S_{j}$ determines the timestamp's validity by verifying whether $\left|T S_{m j}-T S_{p}\right| \leq \triangle T$ holds, where $\triangle T$ denotes expected transmission delay. If the above condition is correct, then $M S_{j}$ executes the next step, otherwise terminates the session.

Step 2. $M S_{j}$ calculates $M G_{6}=h\left(I D_{m j} \| X_{m j}\right), M G_{7}=$ $M G_{3} \oplus M G_{6}=I D_{p}, M G_{8}=h\left(I D_{p} \| X_{m j}\right), M G_{9}=$ $M G_{4} \oplus M G_{7} \oplus M G_{8}=R_{p}, M G_{10}=h\left(M G_{8}\left\|M G_{3}\right\|\right.$ $\left.M G_{4}\left\|M G_{9}\right\| T S_{p}\right) . M S_{j}$ now verifies whether $M G_{10}=$ $? M G_{5}$. If $M G_{10} \neq M G_{5}$, then $M S_{j}$ aborts the session, otherwise authenticates the $U_{p}$. Note that, Das et al.'s protocol follows the same technique used in protocol [29] to mitigate replay and man-in-the-middle attacks.

Step 3. $M S_{j}$ creates a random number $R_{m j}$ and timestamp $T S_{m s}$, and then calculates $M G_{11}=h\left(I D_{m j} \|\right.$ $\left.I D_{p k} \| X_{m k}\right), M G_{12}=I D_{p} \oplus M G_{11}, M G_{13}=$ $h\left(I D_{p} \| X_{m k}\right) \oplus R_{m j}, M G_{14}=I D_{p} \oplus M G_{9} \oplus R_{m j}$, $M G_{15}=h\left(I D_{p}\left\|M G_{11}\right\| M G_{12}\left\|M G_{13}\right\| M G_{14} \|\right.$ $\left.M G_{9} \quad\left\|R_{m j} \quad\right\| T S_{m s}\right) . M S_{j}$ sends a request message $\left\langle I D_{m j}, I D_{p k}, M G_{12}, M G_{13}, M G_{14}, M G_{15}, T S_{m s}\right\rangle$ to $P S_{k}$ over any unreliable network.

Step 4. On receiving $\left\langle I D_{m j}, I D_{p k}, M G_{12}, M G_{13}, M G_{14}\right.$, $\left.M G_{15}, T S_{m s}\right\rangle, P S_{k}$ first checks whether $\left|T S_{m k}-T S_{m s}\right| \leq$ $\triangle T$ holds. If the condition is correct, then $P S_{k}$ proceeds with further operation, otherwise terminates the current session. $P S_{k}$ computes $M G_{16}=h\left(I D_{m j}\left\|I D_{m k}\right\| X_{m k}\right), M G_{17}=$ $M G_{12} \oplus M G_{16}=I D_{p}, M G_{18}=M G_{13} \oplus h\left(M G_{17} \|\right.$ $\left.X_{m k}\right)=R_{m j}, M G_{19}=M G_{14} \oplus M G_{17} \oplus M G_{18}=R_{p}$, $M G_{20}=h\left(M G_{17}\left\|M G_{16}\right\| M G_{12}\left\|M G_{13}\right\| M G_{14} \|\right.$ $\left.M G_{19}\left\|M G_{18}\right\| T S_{m s}\right)$. $P S_{k}$ now checks whether $M G_{20}=$ $M G_{15}$ holds. If $M G_{20} \neq M G_{15}$, then $P S_{k}$ terminates the 
session, otherwise determines that both $U_{p}$ and $M S_{j}$ are authentic entities.

Step 5. $P S_{k}$ creates random number $R_{m k}$, chooses the current timestamp $T S_{k}$, and computes $M G_{21}=h\left(M G_{17} \|\right.$ $\left.X_{m k}\right)=h\left(I D_{p} \| X_{m k}\right), M G_{22}=M G_{17} \oplus M G_{19} \oplus R_{m k}$, $M G_{23}=M G_{21} \oplus R_{m k}, S K_{U_{p}, P S_{k}}=h\left(M G_{17}\left\|I D_{p k}\right\|\right.$ $\left.M G_{19}\left\|R_{m k}\right\| M G_{21} \| T S_{k}\right), M G_{24}=h\left(S K_{U_{p}, P S_{k}} \|\right.$ $\left.M G_{22}\left\|M G_{23}\right\| M G_{19}\left\|R_{m k}\right\| T S_{k}\right)$. Now, $P S_{k}$ sends $\left\langle I D_{m k}, M G_{22}, M G_{23}, M G_{24}, T S_{k}\right\rangle$ to $U_{p}$ via any unreliable network.

Step 6. On receiving $\left\langle I D_{m k}, M G_{22}, M G_{23}, M G_{24}, T S_{k}\right\rangle$, the smartcard of $U_{p}$ chooses a timestamp $T S_{p}^{*}$ and checks whether the condition $\left|T S_{p}^{*}-T S_{k}\right| \leq \triangle T$ is correct. If it is incorrect, then the smartcard of $U_{p}$ aborts the session, otherwise calculates $M G_{25}=M G_{22} \oplus\left(I D_{p} \oplus R_{p}\right)=R_{m k}$, $M G_{26}=M G_{23} \oplus M G_{25}=h\left(I D_{p} \| X_{k}\right), S K_{U_{p}, P S_{k}}^{*}=$ $h\left(I D_{p}\left\|I D_{m k}\right\| R_{p}\left\|M G_{25}\right\| M G_{26} \| T S_{k}\right), M G_{27}=$ $h\left(S K_{U_{p}, P S_{k}}^{*}\left\|M G_{22}\right\| M G_{23}\left\|R_{p}\right\| M G_{25} \| T S_{k}\right)$. Now, the smartcard of $U_{p}$ checks whether $M G_{27}=? M G_{24}$ holds. If $M G_{27} \neq M G_{24}$, then the smartcard of $U_{p}$ terminates the session, otherwise, believes that $P S_{k}$ is authentic entity and the common session session key is $S K_{U_{p}, P S_{k}}^{*}=S K_{U_{p}, P S_{k}}$.

\section{Security Weaknesses in Das et al.'s Protocol}

We will now reveal the insecurity of Das et al.'s protocol.

\section{A. Loss of Patient Anonymity}

As previously mentioned, the anonymity of a patient is highly important [17]. Das et al.'s protocol claimed that the patients are anonymous in their system. However, we will now demonstrate how this property can be violated.

1) Loss of Anonymity using Smartcard Information: In this method, we assume that a legitimate patient can act as a malicious patient or adversary, who has the smartcard of a legitimate patient, and thus can retrieve smartcard information $\left\langle I D_{m j}, A_{m j}, P_{m j}, E_{p}, F_{p}, h(\cdot), \operatorname{Gen}(\cdot), \operatorname{Rep}(\cdot), t\right\rangle$ (e.g. using the technique proposed in [30], [31]). As the malicious patient is legitimate, he/she can compute $h\left(I D_{m j} \| X_{m j}\right)$ as $\left(P_{m j} \oplus P W R_{p}\right)$ using his/her $P W R_{p}$. Since the login information $M G_{3}$ is transmitted through an unreliable network, the malicious patient can intercept $M G_{3}$. Now, the malicious patient can find $I D_{p}$ as $M G_{3} \oplus h\left(I D_{m j} \| X_{m j}\right)$. Thus, Das et al.'s protocol does not ensure patient anonymity.

2) Loss of Anonymity using Login and Authentication Messages: Another legitimate patient may seek to leak medical server information and become a malicious patient (e.g. registering with the server in order to exploit this vulnerability), which we denote as $\mathcal{A}$. In Das et al.'s protocol, the system's information $M G_{11}$ is static and can easily be computed by the legitimate patient as $I D_{p} \oplus M G_{12}$. Note that it is not difficult to make the information $M G_{11}$ public. The malicious user now can compute $I D_{p}$ as $M G_{11} \oplus M G_{12}$ of any legitimate patient only using the login and authentication messages, which are transmitted over an insecure network. In other words, anyone can break the anonymity assurance in Das et al.'s protocol.

\section{B. Loss of Traceability Attack}

For a stronger sense of anonymity, traceability is always regarded as an important property. Now, we demonstrate that Das et al.'s protocol cannot ensure the traceability property. We assume that the adversary $\mathcal{A}$ intercepts two successive login messages $\left\langle I D_{m j}, I D_{p k}, M G_{3}, M G_{4}, M G_{5}, T S_{p}\right\rangle$ and $\left\langle I D_{m j}^{\prime}, I D_{p k}^{\prime}, M G_{3}^{\prime}, M G_{4}^{\prime}, M G_{5}^{\prime}, T S_{p}^{\prime}\right\rangle$, where $M G_{2}=$ $P_{m j} \oplus R P W_{p}^{*}=h\left(I D_{m j} \| X_{m j}\right), M G_{3}=I D_{p} \oplus M G_{2}$. Note that the computation of $M G_{3}$ depends only on the patient's and server's information. If $\left(M G_{3}=? M G_{3}^{\prime}\right)$, then $\mathcal{A}$ believes that the two login messages are from the same patient. Thus, Das et al.'s protocol is not sure against traceability attack.

\section{New Smartcard Launch Attack}

This is another common security feature typically required of smartcards, where an adversary $\mathcal{A}$ attempts to issue new smartcard by using a legitimate patient's identity. In order to launch this attack, the following operations are required to be performed.

Step 1. In Section III-A2, we present the approach to find $I D_{p}$ of $U_{p}$. Therefore, $\mathcal{A}$ performs the same approach to know the $I D_{p}$ of $U_{p}$

Step 2. Now, $\mathcal{A}$ computes $M G_{11}=h\left(I D_{m j} \| X_{m j}\right)=$ $M G_{12} \oplus I D_{p}$.

Step 3. $\mathcal{A}$ chooses a new password $P W_{p}^{n}$, biometric $B_{p}^{n}$ and computes $R P W_{p}^{n}=h\left(I D_{p}\left\|K_{p}^{n}\right\| P W_{p}^{n}\right), A_{m j}^{n}=$ $A_{m j} \oplus R P W_{p}^{*} \oplus R P W_{p}^{n}, P_{m j}^{n}=P_{m j} \oplus R P W_{p}^{*} \oplus R P W_{p}^{n}$, $\operatorname{Gen}\left(B_{p}^{n}\right)=\left(\sigma_{p}^{n}, \tau_{p}^{n}\right), E_{p}^{n}=h\left(\begin{array}{lll}I D_{p} & \| & \sigma_{p}^{n}\end{array}\right) \oplus K_{p}^{n}$, $F_{p}^{n}=h\left(I D_{p}\left\|R P W_{p}^{n}\right\| \sigma_{p}^{n}\right)$, where $R P W_{p}^{*}$ can be computed as discussed in Section III-A1. Finally, $\mathcal{A}$ stores $\left\langle I D_{m j}, A_{m j}^{n}, P_{m j}^{n}, E_{p}^{n}, F_{p}^{n}, h(\cdot), \operatorname{Gen}(\cdot), \operatorname{Rep}(\cdot), t\right\rangle$ into the new smartcard, where $1 \leq j \leq m+m^{\prime}$. Thus, $\mathcal{A}$ can launch the above attack successfully.

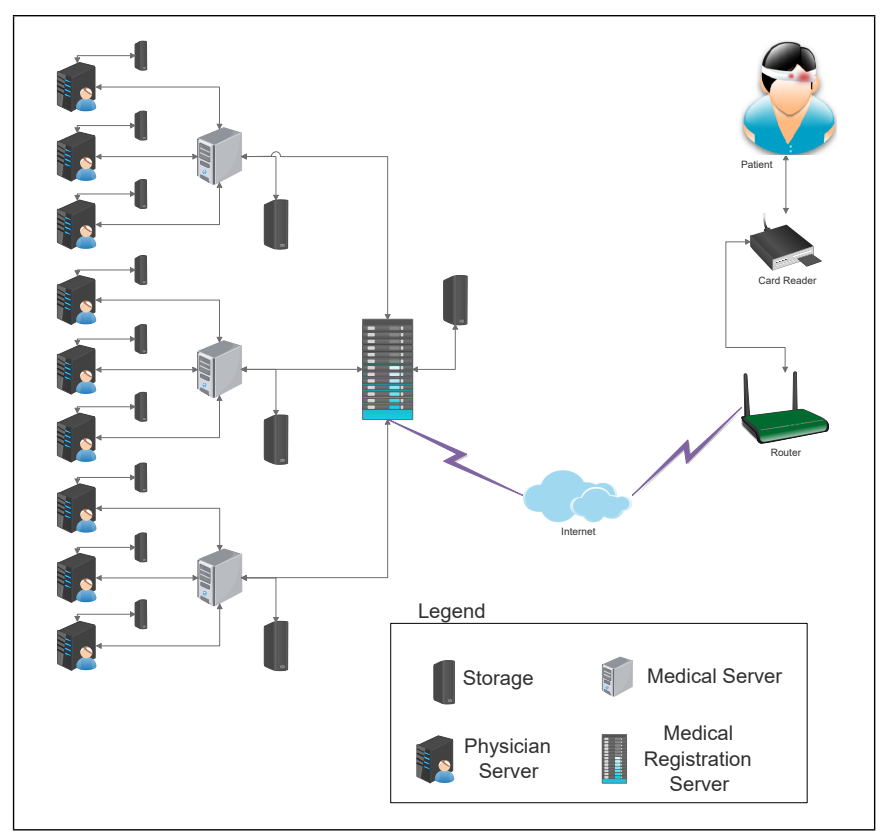

Fig. 1. Multi-medical system framework [17] 


\section{Proposed PROTOCOL}

Fig. 1 outlines the medical system architecture proposed in [17], which comprises medical registration server, medical server, physician server and patients. The medical registration server is mainly responsible for providing registration service to all medical servers. In this architecture, there are several medical servers located in different locations for providing registration services to the physician servers and the patients. Note that the physician servers may be under the control of any medical server. In addition, it participates in the negotiation of session key between a physician server and patient. The patients may seek medical treatment from anywhere and anytime. After performing the registration with a medical server, the patient logins into the system to negotiate a session key with a physician server. This session key will be used to exchange some medical information of the patient.

Our proposed protocol consists of nine phase, namely: setup, medical-server registration, physician-server registration, patient registration, login, authentication and session key negotiation, new physician-server addition, password renewal, and biometric renewal. Table I summarizes the notations used in the protocol.

TABLE I

NOTATIONS

\begin{tabular}{|ll|}
\hline Symbol & Description \\
\hline$M R S$ & Central-medical-registration-server \\
$U_{p}$ & Patient, $(1 \leq p \leq m)$ \\
$M S_{j}$ & Medical-server, $(1 \leq j \leq n)$ \\
$P S_{k}$ & Physician-server, $(1 \leq k \leq l)$ \\
$I D_{m j}$ & Identity of the $M S_{j}$ \\
$I D_{p k}$ & Identity of the $P S_{k}$ \\
$I D_{p}$ & Identity of the $U_{p}$ \\
$T I D_{p}$ & Temporary unique identity of the $U_{p}$ \\
$B_{p}$ & Biometric of the $U_{p}$ \\
$P W_{p}$ & Password of the $U_{p}$ \\
$M K_{s h r d}$ & Common key between all the $M S_{j}$ \\
$S K$ & Session key of the protocol \\
$H(\cdot)$ & Bio-hashing function \\
$h(\cdot)$ & Hash function $\{0,1\}^{*} \rightarrow\{0,1\}^{k}$ \\
$\|$ & Concatenation operation $(a \| b)$ \\
$\oplus$ & Bitwise XOR operation $(a \oplus b)$ \\
\hline
\end{tabular}

\section{A. Setup Phase}

$M R S$ (medical registration server) selects a master key $M K_{\text {shrd }}$ and shares it among all medical servers $M S_{j}(1 \leq$ $j \leq n$ ) securely. Note that, this key cannot be compromised under any circumstances.

\begin{tabular}{|c|c|}
\hline Medical server $M S_{j}$ & Medical registration server $M R S$ \\
\hline \multicolumn{2}{|l|}{$\begin{array}{l}\text { Chooses an unique identity } I D_{m j} \\
\qquad\left\langle I D_{m j}\right\rangle\end{array}$} \\
\hline \multicolumn{2}{|l|}{ (via a secure channel) } \\
\hline & $\begin{array}{l}\text { Compute } M S_{\text {key }_{j}}=h\left(I D_{m j} \| M S K\right) \\
\text { Store }\left\langle I D_{m j}, E_{M S K}\left(M S_{k e y_{j}}\right)\right\rangle \text { in the table } \\
\qquad\left\langle M S_{k e y_{j}}\right\rangle\end{array}$ \\
\hline & (via a secure channel) \\
\hline Keep $M S_{k e y_{j}}$ secret & \\
\hline
\end{tabular}

Fig. 2. Medical server registration phase of our protocol.

\section{B. Medical Server Registration Phase}

Each medical server $M S_{j}(1 \leq j \leq n)$ needs to register with $M R S$ before accessing any service. $M S_{j}$ chooses an unique identity $I D_{m j}$ and sends it to $M R S$ via a secure channel. On receiving $I D_{m j}, M R S$ calculates $M S_{k e y_{j}}=$ $h\left(I D_{m j} \| M S K\right)$ as a secret key of $M S_{j}$ and stores $\left\langle I D_{m j}, E_{M S K}\left(M S_{k e y_{j}}\right)\right\rangle$ in the table for all $M S_{j}(1 \leq j \leq$ $n)$. Finally, $M R S$ sends $\left\langle M S_{k e y_{j}}\right\rangle$ to $M S_{j}$ via a secure channel. Note that, $M S_{j}$ keeps $M S_{k e y_{j}}$ secret and none can obtain it by any means. Fig. 2 also describes this phase.

\begin{tabular}{|ll|}
\hline Physician server $P S_{k}$ & Medical server $M S_{j}$ \\
\hline $\begin{array}{l}\text { Chooses an unique identity } I D_{p k} \\
\left\langle I D_{p k}\right\rangle\end{array}$ & \\
\hline (via a secure channel) & Compute $P S_{k e y_{k}}=h\left(I D_{p k} \| M S_{k e y_{j}}\right)$ \\
& $\langle$ (via a secure channel) \\
Keep $P S_{\text {key }}$ secret & \\
\hline
\end{tabular}

Fig. 3. Physician server registration phase of our protocol.

\section{Physician Server Registration Phase}

The main objective of this phase (see Fig. 3) is to negotiate a session key between the patient $U_{p}$ and a physician server $P S_{k}$, which is managed by a doctor. Therefore, all physician servers $P S_{k}(1 \leq k \leq l)$ must register with the home medical server $M S_{j}$. Specifically, $P S_{k}$ chooses a unique identity $I D_{p k}$ and sends it to $M S_{j}$ via a secure channel. On getting $I D_{p k}$, $M S_{j}$ calculates $P S_{k e y_{k}}=h\left(I D_{p k} \| M S_{k e y_{j}}\right)$ and sends to $P S_{k}$ securely.

\begin{tabular}{|c|c|}
\hline Patient $U_{p}$ & Medical-server $M S_{j}$ \\
\hline \multicolumn{2}{|l|}{$\begin{array}{c}\text { Chooses an unique identity } I D_{p} \\
\left\langle I D_{p}\right\rangle\end{array}$} \\
\hline \multicolumn{2}{|l|}{ (via a secure channel) } \\
\hline & $\begin{array}{l}\text { Generate a random number } r_{p} \\
\text { Select an unique temporary identity } T I D_{p} \\
\text { Compute } A_{p}=h\left(I D_{p}\left\|r_{p}\right\| M S_{k e y_{j}}\right) \\
\text { Store }\left\langle A_{p}, I D_{p}, T I D_{p}\right\rangle \text { in the smartcard } \\
\text { Keep secret }\left\langle T I D_{p}, E_{M S_{\text {key }}}\left(I D_{p}\right), r_{p}\right\rangle \\
\quad\langle\text { Smartcard }\rangle\end{array}$ \\
\hline & (via a secure channel) \\
\hline \multicolumn{2}{|l|}{ Enter $I D_{p}, P W_{p}$ and $B_{p}$ into the smartcard } \\
\hline \multicolumn{2}{|l|}{ Compute $D_{p}=H\left(B_{p}\right)$} \\
\hline \\
\hline \multicolumn{2}{|l|}{ Compute $E_{p}=h\left(I D_{p}\left\|P W_{p}\right\| D_{p}\right)$} \\
\hline \multicolumn{2}{|l|}{ Delete $A_{p}$ from the smartcard } \\
\hline Store $\left\langle C_{p}, D_{p}, E_{p}, I D_{p}, T I D_{p}, h(\cdot), H(\cdot)\right\rangle$ & \\
\hline
\end{tabular}

Fig. 4. Patient registration phase of our protocol.

\section{Patient Registration Phase}

In this phase (see Fig. 4), a patient $U_{p}(1 \leq p \leq m)$ needs to be registered with a nearby medical server $M S_{j}$ in order to access physician servers located in different places.

Step 1. $U_{p}$ chooses a unique identity $I D_{p}$ and sends it to $M S_{j}$ securely.

Step 2. $M S_{j}$ computes $A_{p}=h\left(I D_{p}\left\|r_{p}\right\| M S_{k e y_{j}}\right)$ and stores $\left\langle A_{p}, I D_{p}, T I D_{p}\right\rangle$ it in the memory of a smartcard, where $r_{p}$ is the random number selected by $M S_{j}$ and $T I D_{p}$ is the unique temporary identity for $U_{p}$. Then, $M S_{j}$ keeps a secret table, which includes a tuple of the form 
$\left\langle T I D_{p}, E_{M S_{k e y}}\left(I D_{p}\right), r_{p}\right\rangle$ for each $U_{p}(1 \leq p \leq m)$. Finally, $M S_{j}$ delivers the smartcard to the $U_{p}$ through an off-line mode.

Step 3. On receiving the smartcard, $U_{p}$ enters his/her identity $I D_{p}$, password $P W_{p}$, and biometric $B_{p}$ into the smartcard. Now, the smartcard calculates $D_{p}=H\left(B_{p}\right), C_{p}=A_{p} \oplus$ $h\left(P W_{p} \| D_{p}\right)$, and $E_{p}=h\left(I D_{p}\left\|P W_{p}\right\| D_{p}\right)$, where $H(\cdot)$ is the bio-hashing function [8].

Step 4. $U_{p}$ deletes $A_{p}$ from the smartcard. Finally, the smartcard holds $\left\langle C_{p}, D_{p}, E_{p}, I D_{p}, T I D_{p}, h(\cdot), H(\cdot)\right\rangle$. The

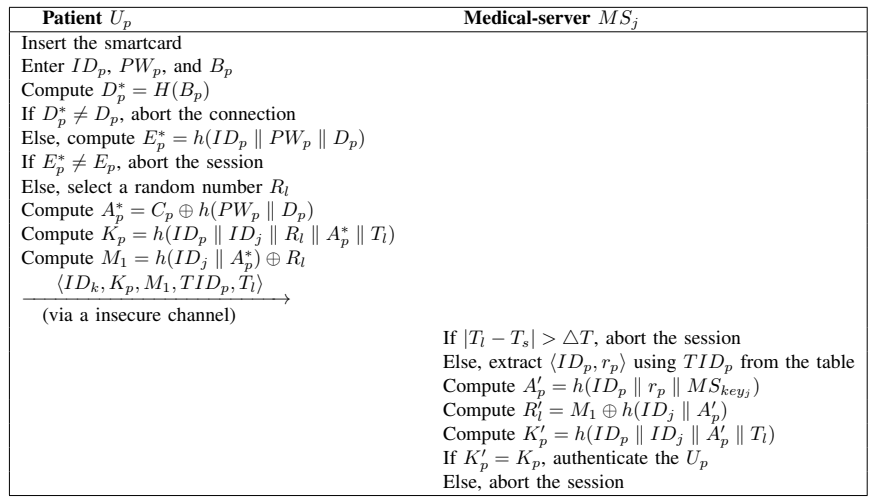

Fig. 5. Login and authentication phase of our protocol.

\section{E. Login and Authentication Phase}

This phase (Fig. 5) is invoked whenever a patient needs to access medical data from a physician server. To complete this phase, the smartcard generates login message on inputting patient information and then forwards the message to $M S_{j}$, where he/she has executed the registration phase.

Step 1. At the beginning, $U_{p}$ inserts the smartcard into the terminal, and enters $I D_{p}, P W_{p}$, and $B_{p}$. Then, the smartcard computes $D_{p}^{*}=H\left(B_{p}\right)$. If $D_{p}^{*} \neq D_{p}$, then the smartcard of $U_{p}$ aborts the session, otherwise calculates $E_{p}^{*}=h\left(I D_{p} \|\right.$ $\left.P W_{p} \| D_{p}\right)$. If $E_{p}^{*} \neq E_{p}$, then the smartcard of the $U_{p}$ aborts the session, otherwise, executes further operations.

Step 2. The smartcard of $U_{p}$ selects a random number $R_{l}$, and timestamp $T_{l}$. The smartcard of $U_{p}$ computes $A_{p}^{*}=C_{p} \oplus$ $h\left(P W_{p} \| D_{p}\right), K_{p}=h\left(I D_{p}\left\|I D_{j}\right\| R_{l}\left\|A_{p}^{*}\right\| T_{l}\right), M_{1}=$ $h\left(I D_{j} \| A_{p}^{*}\right) \oplus R_{l}$.

Step 3. Now, the smartcard of $U_{p}$ provides a option to choose the identity $I D_{k}$ of the physician server $P S_{k}$, from which the patient wishes to access the service. The smartcard then sends $\left\langle I D_{k}, K_{p}, M_{1}, T I D_{p}, T_{l}\right\rangle$ to $M S_{j}$ through an unreliable network.

Step 4. Whenever $M S_{j}$ receives the login message $\left\langle I D_{k}, K_{p}, M_{1}, T I D_{p}, T_{l}\right\rangle$, it checks whether $\left|T_{l}-T_{s}\right| \leq \triangle T$ holds, where $T_{s}$ is current timestamp. If it holds, then $M S_{j}$ extracts $\left\langle I D_{p}, r_{p}\right\rangle$ from the table using $T I D_{p}$ and calculates $A_{p}^{\prime}=h\left(I D_{p}\left\|r_{p}\right\| M S_{k e y_{j}}\right), R_{l}^{\prime}=M_{1} \oplus h\left(I D_{j} \| A_{p}^{\prime}\right)$, $\stackrel{p}{K_{p}^{\prime}}=h\left(I D_{p}\left\|I D_{j}\right\| A_{p}^{\prime} \| T_{l}\right)$. If $K_{p}^{\prime} \neq K_{p}$, then $M S_{j}$ aborts the session, otherwise, authenticates the $U_{p}$. The

\section{F. Session Key Agreement Phase}

This phase (Fig. 6 ) is used to execute the mutual authentication and session between the entities in the protocol execution.
Step 1. After the execution of the login phase, $M S_{j}$ receives the login message with $I D_{k}$ as the parameter. In our protocol, the execution of Cases 1 and 2 depends on the existence of $I D_{p}$. If $I D_{k}$ is present in the local database of $M S_{j}$, then Case 1 will be executed to allow the patient to negotiate a session key between $U_{p}$ and $P S_{k}$. Here, $I D_{k}$ is the identity of $P S_{k}$. On the other hand, if $I D_{k}$ is not found in the local database, then $M S_{j}$ searches in the global database. If $M S_{j}$ is found, then we have Case 2.

\section{Case 1.}

Step 1-1. $M S_{j}$ calculates $P S_{\text {key }}=h\left(I D_{k} \| M S_{k e y_{j}}\right), L_{p}=$ $h\left(I D_{k}\left\|T I D_{p}\right\| P S_{\text {key }_{k}}\right) \oplus R_{l}^{\prime}, N_{p}=h\left(T I D_{p} \| P S_{\text {key }_{k}}\right) \oplus$ $I D_{p}, O_{p}=h\left(I D_{p}\left\|I D_{j}\right\| I D_{k}\left\|R_{l}^{\prime}\right\| T_{s}\right)$ and then sends $\left\langle L_{p}, N_{p}, O_{p}, I D_{j}, T_{s}, T I D_{p}\right\rangle$ to the $P S_{k}$ over an unreliable network.

Step 1-2. On receiving $\left\langle L_{p}, N_{p}, O_{p}, I D_{j}, T_{s}, T I D_{p}\right\rangle, P S_{k}$ first checks whether $\left|T_{s}-T_{k}\right| \leq \triangle T$ holds, where $T_{k}$ is the current timestamp. If the above verification fails, then $P S_{k}$ terminates the connection, otherwise computes $R_{l}^{\prime \prime}=L_{p} \oplus$ $h\left(I D_{k}\left\|T I D_{p}\right\| P S_{\text {key }_{k}}\right), I D_{p}^{\prime}=N_{p} \oplus h\left(T I D_{p} \| P S_{\text {key }}\right)$, and $O_{p}^{\prime \prime}=h\left(I D_{p}^{\prime}\left\|I D_{j}\right\| I D_{k}\left\|R_{l}^{\prime \prime}\right\| T_{s}\right)$. If $O_{p}^{\prime \prime} \neq O_{p}$, then $P S_{k}$ aborts the connection, otherwise executes further computations.

Step 1-3. $P S_{k}$ now selects a random number $R_{p}$, a session key $S K$ randomly, and then calculates $P_{p}=h\left(I D_{p}\|S K\|\right.$ $\left.I D_{k}\left\|T_{k}\right\| h\left(I D_{p} \| P S_{k e y_{k}}\right)\right), Q_{p}=R_{l}^{\prime \prime} \oplus h\left(I D_{p} \|\right.$ $\left.P S_{\text {keyk }}\right), S_{p}=S K \oplus h\left(I D_{p}\left\|R_{l}^{\prime \prime}\right\| T_{k}\right)$. Finally, $P S_{k}$ sends $\left\langle P_{p}, Q_{p}, S_{p}, T_{k}\right\rangle$ to $U_{p}$ via an unreliable network.

Step 1-4. $U_{p}$ first takes whether $\left|T_{t}-T_{k}\right| \leq \triangle T$ holds, where $T_{t}$ is the current timestamp. If the above condition is correct, then $U_{p}$ extracts $h\left(I D_{p} \| P S_{\text {key }_{k}}\right)$ using $R_{l}$ and $S K^{\prime}$ and $\left\langle I D_{p}, R_{l}\right\rangle$ from $Q_{p}$ and $S_{p}$, respectively. Now, $U_{p}$ further calculates $P_{p}^{\prime}=h\left(I D_{p}\left\|S K^{\prime}\right\| I D_{k}\left\|T_{k}\right\|\right.$ $\left.h\left(I D_{p} \| P S_{\text {keyk }}\right)\right)$. If $P_{p}^{\prime}=P_{p}$, then $U_{p}$ authenticates the $P S_{k}$, otherwise terminates the session.

Step 1-5. In order to resist traceability attack, $U_{p}$ sends an acknowledgment to $M S_{j}$ that the session is correctly executed. On receiving the acknowledgment, $M S_{j}$ generates a random unique identity $T I D_{p}^{\prime} \neq T I D_{p}$ and computes $M_{2}=T I D_{p}^{\prime} \oplus$ $R_{l}, M_{3}=h\left(I D_{p}\left\|T I D_{p}^{\prime}\right\| R_{l}\right)$, and sends $\left\langle M_{2}, M_{3}\right\rangle$ to $U_{p}$ over an unreliable network.

Step 1-6. On receiving $\left\langle M_{2}, M_{3}\right\rangle, U_{p}$ extracts $T I D_{p}^{\prime}$ as $T I D_{p}^{\prime}=M_{2} \oplus R_{l}$ and $M_{3}^{\prime}=h\left(I D_{p}\left\|T I D_{p}^{\prime}\right\| R_{l}\right)$. If the condition $M_{3}^{\prime}=M_{3}$ is correct, then the smartcard of $U_{p}$ replaces $T I D_{p}$ with $T I D_{p}^{\prime}$ in the memory.

Case 2.

In this case, $M S_{j}$ extracts the identity $I D_{m j}$ of $M S_{j}$, which is managed by the the physician server $P S_{k}$ with identity $I D_{p k}$. $M S_{j}$ executes the following steps to negotiate a secure session key between $U_{j}$ and $P S_{k}$.

Step 2-1. The home medical server $M S_{j}$ computes $Y_{1}=$ $I D_{p} \oplus h\left(I D_{m j}^{\prime}\left\|T_{1}\right\| M K_{s h r d}\right)$ and $Y_{2}=h\left(I D_{m j} \|\right.$ $\left.I D_{m j}^{\prime}\left\|M K_{s h r d}\right\| T_{1}\right)$, where $I D_{m j}^{\prime}$ is the identity of the foreign medical server $M S_{j}$. Finally, $M S_{j}$ sends $\left\langle Y_{1}, Y_{2}, T_{1}, I D_{m j}\right\rangle$ through an insecure network to $M S_{j}$, which is under the control of $P S_{k}$.

Step 2-2. On receiving $\left\langle Y_{1}, Y_{2}, T_{1}, I D_{j}\right\rangle, M S_{j}$ first checks whether $\left|T_{1}-T_{2}\right| \leq \triangle T$ holds. If the verification fails, then 


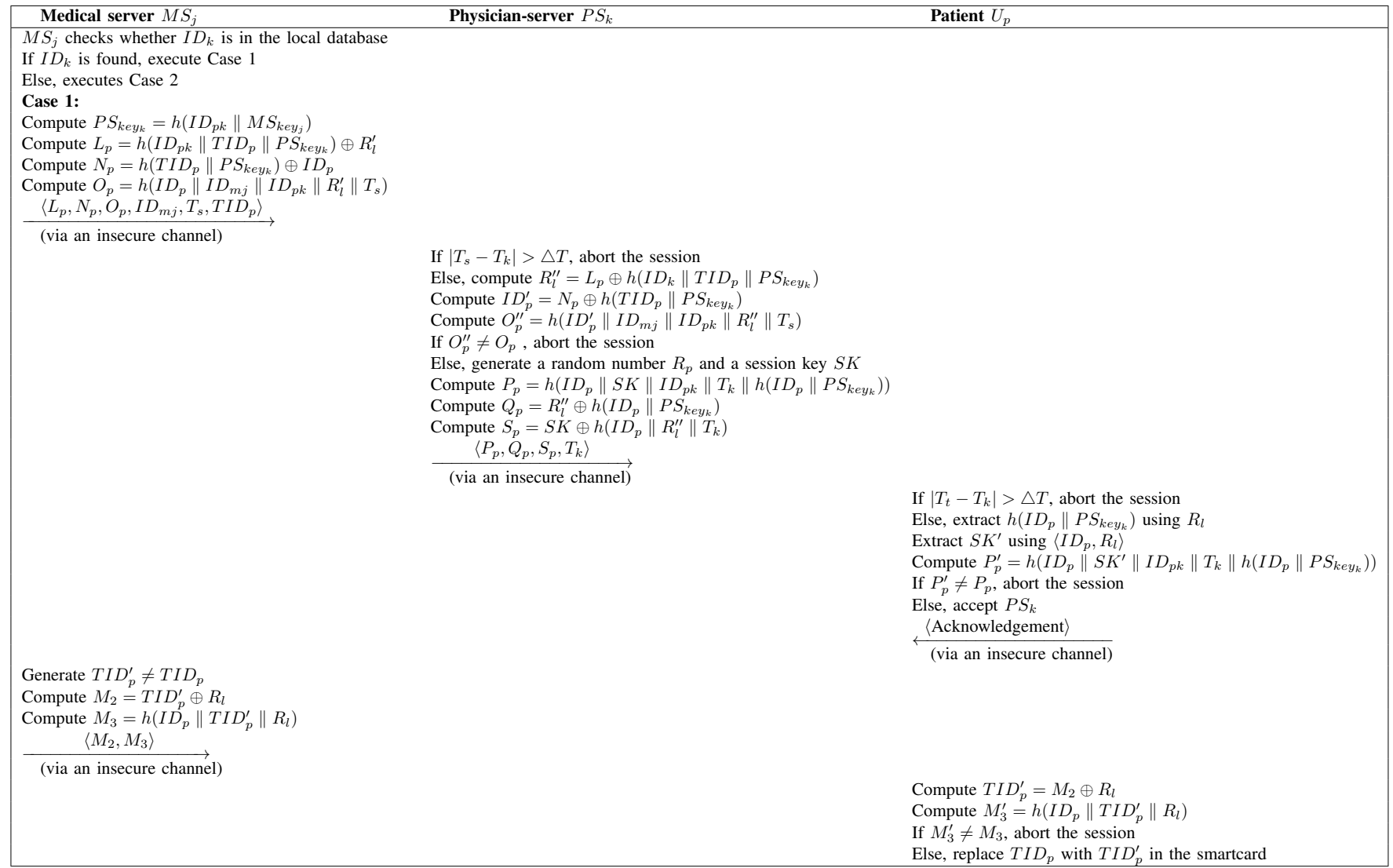

Fig. 6. Session key agreement phase of our protocol (Case 1).

\begin{tabular}{|c|c|c|c|}
\hline Home medical server $M S_{j}$ & Foreign medical server $M S_{j}$ & Physician server $P S_{k}$ & Patient $U_{p}$ \\
\hline \multicolumn{4}{|l|}{$\begin{array}{l}\text { Assume that } I D_{p k} \text { is found in the global database } \\
\text { Compute } Y_{1}=I D_{p} \oplus h\left(I D_{m j}^{\prime}\left\|T_{1}\right\| M K_{s h r d}\right) \\
\text { Compute } Y_{2}=h\left(I D_{m j}\left\|I D_{m j}^{\prime}\right\| M K_{s h r d} \| T_{1}\right) \\
\left.\qquad Y_{1}, Y_{2}, T_{1}, I D_{m j}\right\rangle\end{array}$} \\
\hline \multirow{4}{*}{ (via a insecure channel) } & & & \\
\hline & $\begin{array}{l}\text { If }\left|T_{1}-T_{2}\right|>\Delta T \text {, aborts the session } \\
\text { Else, compute } Y_{2}^{\prime}=h\left(I D_{m j}\left\|I D_{m j}^{\prime}\right\| M K_{s h r d} \| T_{1}\right) \\
\text { If } Y_{2}^{\prime} \neq Y_{2} \text {, aborts the session } \\
\text { Else, extract } I D_{p}=Y_{1} \oplus h\left(I D_{m j}^{\prime}\left\|T_{1}\right\| M K_{s h r d}\right) \\
\text { Compute } Y_{3}=h\left(I D_{p k}\left\|I D_{p}\right\| T_{1} \| P S_{\text {key }_{j}}\right) \\
\text { Compute } Y_{4}=I D_{p} \oplus h\left(I D_{p k}\left\|P S_{k e y_{j}}\right\| T_{1}\right) \\
\qquad\left\langle Y_{3}, Y_{4}, T_{2}\right\rangle\end{array}$ & & \\
\hline & (via a insecure channel) & $\begin{array}{l}\text { If }\left|T_{2}-T_{3}\right|>\Delta T \text { aborts the session } \\
\text { Else, generate a random session key } S K \\
\text { Compute } I D_{p}^{\prime}=Y_{4} \oplus h\left(I D_{p k}\left\|P S_{\text {keyj }}\right\| T_{1}\right) \\
\text { Compute } Y_{3}^{\prime}=h\left(I D_{p k}\left\|I D_{p}^{\prime}\right\| T_{1} \| P S_{\text {key }_{j}}\right) \\
\text { If }\left(Y_{3}^{\prime} \neq Y_{3}\right) \text {, abort the session } \\
\text { Compute } Y_{5}=I D_{p}^{\prime} \oplus S K \\
\text { Compute } Y_{6}=h\left(I D_{p}^{\prime}\|S K\| I D_{p k} \| T_{3}\right) \\
\left.\qquad Y_{5}, y_{6}, T_{3}\right\rangle\end{array}$ & \\
\hline & & (via a insecure channel) & $\begin{array}{l}\text { If }\left|T_{3}-T_{4}\right|>\Delta T, \text { aborts the session } \\
\text { Else, compute } Y_{6}^{\prime}=h\left(I D_{p}\|S K\| I D_{p k} \| T_{3}\right) \\
\text { If } Y_{6}^{\prime} \neq=Y_{6}, \text { the } U_{p} \text { abort the session } \\
\text { \{ckknowledgement })\end{array}$ \\
\hline $\begin{array}{l}\text { Receives acknowledgment } \\
\text { Generates } T I D_{p}^{\prime} \text { where } T I D_{p}^{\prime} \neq T I D_{p} \\
\text { Computes } M_{22}=T I D_{p}^{\prime} \oplus R_{l} \\
\text { Computes } M_{33}=h\left(I D_{p}\left\|T I D_{p}^{\prime}\right\| R_{l}\right) \\
\quad\left\langle M_{22}, M_{33}\right\rangle\end{array}$ & & & (via a insecure channel) \\
\hline (via a insecure channel) & & & $\begin{array}{l}\text { Receives messages from } M S_{j} \\
\text { Computes } T I D_{p}^{\prime}=M_{22} \oplus R_{l} \\
\text { Computes } M_{33}^{\prime}=h\left(I D_{p}\left\|T I D_{p}^{\prime}\right\| R_{l}\right) \\
\text { If } \mid M_{33}^{\prime} \neq M_{33}, \text { aborts the session } \\
\text { replaces } T I D_{p} \text { with } T I D_{p}^{\prime} \text { in the smartcard }\end{array}$ \\
\hline
\end{tabular}

Fig. 7. Session key agreement phase of our protocol (Case 2).

$M S_{j}$ aborts the session, otherwise, computes $Y_{2}^{\prime}=h\left(I D_{m j} \|\right.$ $\left.I D_{m j}^{\prime}\left\|M K_{s h r d}\right\| T_{1}\right)$. If $\left(Y_{2}^{\prime} \neq Y_{2}\right)$, then $M S_{j}$ aborts the connection, otherwise extracts $I D_{p}=Y_{1} \oplus h\left(I D_{m j}^{\prime} \|\right.$ $\left.T_{1} \| M K_{s h r d}\right)$, and computes $Y_{3}=h\left(I D_{p k}\left\|I D_{p}\right\| T_{1} \|\right.$ 
$\left.P S_{k e y_{j}}\right), Y_{4}=I D_{p} \oplus h\left(I D_{p k}\left\|P S_{k e y_{j}}\right\| T_{1}\right) . M S_{j}$ sends $\left\langle Y_{3}, Y_{4}, T_{2}\right\rangle$ to the $P S_{k}$ through an insecure network.

Step 2-3. On receiving $\left\langle Y_{3}, Y_{4}, T_{2}\right\rangle, P S_{k}$ first checks whether $\left|T_{2}-T_{3}\right| \leq \triangle T$ holds, where $T_{3}$ is the current timestamp. If the timestamp verification fails, then $P S_{k}$ aborts the session, otherwise, generates a random session key $S K$ and computes $I D_{p}^{\prime}=Y_{4} \oplus h\left(I D_{p k}\left\|P S_{k e y_{j}}\right\| T_{1}\right), Y_{3}^{\prime}=h\left(I D_{k}\left\|I D_{p}^{\prime}\right\|\right.$ $\left.T_{1} \| P S_{\text {key }}\right)$. If $Y_{3}^{\prime} \neq Y_{3}$, then $P S_{k}$ aborts the connection, otherwise, computes $Y_{5}=I D_{p}^{\prime} \oplus S K, Y_{6}=h\left(I D_{p}^{\prime}\|S K\|\right.$ $\left.I D_{p k} \| T_{3}\right)$ and sends $\left\langle Y_{5}, Y_{6}, T_{3}\right\rangle$ to $U_{p}$ through an insecure network.

Step 2-4. $U_{p}$ checks whether $\left|T_{3}-T_{4}\right| \leq \triangle T$ holds, where $T_{4}$ is the current timestamp. If the timestamp verification fails, then $U_{p}$ aborts the session, otherwise, computes $Y_{6}^{\prime}=$ $h\left(I D_{p}\|S K\| I D_{p k} \| T_{3}\right)$. If $Y_{6}^{\prime} \neq Y_{6}$, then $U_{p}$ aborts the connection, otherwise, sends an acknowledgment to the home medical server $M S_{j}$ to confirm that the session key is negotiated with the correct $P S_{k}$.

Step 2-5. On receiving the acknowledgment, $M S_{j}$ generates a new random unique identity $T I D_{p}^{\prime} \neq T I D_{p}$ and computes $M_{22}=T I D_{p}^{\prime} \oplus R_{l}, M_{33}=h\left(I D_{p}\left\|T I D_{p}^{\prime}\right\| R_{l}\right)$, and sends $\left\langle M_{22}, M_{33}\right\rangle$ to $U_{p}$ over an unreliable network.

Step 2-6. On receiving $\left\langle M_{22}, M_{33}\right\rangle, U_{p}$ extracts $T I D_{p}^{\prime}$ as $T I D_{p}^{\prime}=M_{22} \oplus R_{l}$ and $M_{33}^{\prime}=h\left(I D_{p}\left\|T I D_{p}^{\prime}\right\| R_{l}\right)$. If the condition $M_{33}^{\prime}=M_{33}$ is correct, then the smartcard of the $U_{p}$ replaces $T I D_{p}$ with $T I D_{p}^{\prime}$ in the memory.

Fig. 7 also outlines this phase.

\section{G. New Physician Server Addition Phase}

It is also necessary to add new physician server(s) to the system for scalability and achieve higher system reliability. Thus, our protocol supports the addition of new physician server. The new physician server $P S_{t}$ first chooses a unique identity $I D_{p t}$ and then sends it to $M S_{j}$ securely. Then, $M S_{j}$ performs $P S_{\text {keyt }}=h\left(I D_{p t} \| M S_{k e y_{j}}\right)$ and sends it to $P S_{t}$ through a secure network. Accordingly, our protocol can increase the flexibility of the system by adding new physician server.

\section{H. Password Update Phase}

The password $P W_{p}$ of patient $U_{p}$ can be updated (periodically).

Step 1. $U_{p}$ inserts the smartcard to the terminal and enters $I D_{p}, P W_{p}$, and $B_{p}$. Then, the smartcard computes $D_{p}^{*}=$ $H\left(B_{p}\right)$. If $D_{p}^{*} \neq D_{p}$, then the smartcard aborts the session, otherwise, computes $E_{p}^{*}=h\left(I D_{p}\left\|P W_{p}\right\| D_{p}\right)$. If $E_{p}^{*} \neq E_{p}$, then the smartcard aborts the session, otherwise requests to enter a new password.

Step 2. Suppose that $U_{p}$ inputs a new password $P W_{p}^{\text {new }}$. The smartcard now performs $A_{p}=C_{p} \oplus h\left(P W_{p} \| D_{p}\right), C_{p}^{\text {new }}=$ $A_{p} \oplus h\left(P W_{p}^{\text {new }} \| D_{p}\right), E_{p}^{\text {new }}=h\left(I D_{p}\left\|P W_{p}^{\text {new }}\right\| D_{p}\right)$ and finally includes $\left\langle C_{p}^{\text {new }}, E_{p}^{\text {new }}\right\rangle$ into the memory and drops $\left\langle C_{p}, E_{p}\right\rangle$.

\section{Biometric Renewal Phase}

This phase updates the biometric information of the registered patient. First, executes Step 1 in Section IV-H to verify the correctness of $I D_{p}, P W_{p}$, and $B_{p}$. If all of these are correct, then the smartcard requests to enter new biometric information. Suppose that $U_{p}$ enters $B_{p}^{\text {new }}$ as the new biometric information. Now the smartcard computes $D_{p}^{\text {new }}=H\left(B_{p}^{\text {new }}\right)$, $C_{p}^{\text {new }}=A_{p} \oplus h\left(P W_{p} \| D_{p}^{\text {new }}\right)$, and $E_{p}^{\text {new }}=h\left(I D_{p} \|\right.$ $\left.P W_{p}^{\text {new }} \| D_{p}^{\text {new }}\right)$ and includes $\left\langle D_{p}^{\text {new }}, C_{p}^{\text {new }}, E_{p}^{\text {new }}\right\rangle$ by replacing the old information $\left\langle D_{p}, C_{p}, E_{p}\right\rangle$.

\section{SECURITy AnALYSis}

This section evaluates the security of the proposed protocol.

\section{A. Capabilities of the Adversary}

In this section, we analyze that whether an adversary can launch attacks or not based on our protocol information under the assumption mentioned in [21]. At first, our objective is to examine whether the adversary could calculate private information or not from the public information. The following cases will demonstrate the above objectives.

- Case 1: We assume that an adversary $\mathcal{A}$ can get the smartcard of the $U_{p}$ and easily extract confidential information to break the security of the proposed protocol by using the processes described in [30], [31]. In our protocol, the smartcard includes $\left\langle C_{p}, D_{p}, E_{p}, I D_{j}, T I D_{p}, h(\cdot), H(\cdot)\right\rangle$, where $D_{p}=H\left(B_{p}\right), C_{p}=A_{p} \oplus h\left(P W_{p} \| D_{p}\right)$, and $E_{p}=h\left(I D_{p}\left\|P W_{p}\right\| D_{p}\right), A_{p}=h\left(I D_{p}\left\|r_{p}\right\|\right.$ $\left.M S_{\text {key }_{j}}\right)$. The objective of the $\mathcal{A}$ is to calculate the private information $\left\langle I D_{p}, P W_{p}, A_{p}\right\rangle$ using the information obtained from the smartcard. Though $\mathcal{A}$ knows $D_{p}$, it is infeasible to calculate $I D_{p}, P W_{p}$ from the known information $E_{p}$ owing to the difficulties of the inversion of the hash function $h(\cdot)$. In the similar fashion, the $\mathcal{A}$ is not able to find $A_{p}$ from $C_{p}$. Therefore, our proposed system is still secure even if the smartcard of the $U_{p}$ is available to the $\mathcal{A}$.

- Case 2. In our protocol, the smartcard sends a login information $\left\langle I D_{p k}, K_{p}, M_{1}, T I D_{p}, T_{l}\right\rangle$ to the $M S_{j}$ through a public network. If $\mathcal{A}$ monitors the network, he/she can easily intercept all these information, where $A_{p}^{*}=C_{p} \oplus h\left(P W_{p} \| D_{p}\right), K_{p}=h\left(I D_{p}\left\|I D_{m j}\right\| R_{l} \|\right.$ $\left.A_{p}^{*} \| T_{l}\right), M_{1}=h\left(I D_{m j} \| A_{p}^{*}\right) \oplus R_{l}$. In this case, the confidential information are $\left\langle A_{p}, I D_{p}, P W_{p}, R_{l}\right\rangle$. In our protocol, we use a one-way hash function $h(\cdot)$ to compute $K_{p}$ and $M_{1}$. All the information $\left\langle A_{p}, I D_{p}, P W_{p}, R_{l}\right\rangle$ are protected due to the $h(\cdot)$, and therefore the $\mathcal{A}$ cannot find these confidential information. Thus, if the $\mathcal{A}$ obtains the message $\left\langle I D_{k}, K_{p}, M_{1}, T I D_{p}, T_{l}\right\rangle$ from the public network, he/she cannot break the security of the proposed system.

- Case 3. In our protocol, the $M S_{j}$ sends the message $\left\langle L_{p}, N_{p}, O_{p}, I D_{m j}, T_{s}, T I D_{p}\right\rangle$ to the $P S_{k}$ through an unreliable network. Therefore, the $\mathcal{A}$ can capture all these information, where $P S_{k e y_{k}}=h\left(I D_{p k} \| M S_{k e y_{j}}\right)$, $L_{p}=h\left(I D_{p k}\left\|T I D_{p}\right\| P S_{\text {key }_{k}}\right) \oplus R_{l}^{\prime}, N_{p}=$ $h\left(T I D_{p} \| P S_{k e y_{k}}\right) \oplus I D_{p}, O_{p}=h\left(I D_{p}\left\|I D_{m j}\right\|\right.$

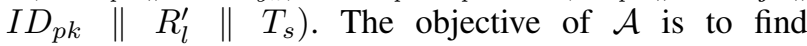
$\left\langle P S_{k e y_{k}}, R_{l}, I D_{p}\right\rangle$. Though the $\mathcal{A}$ knows $I D_{p k}$, however, he/she is not able to compute $P S_{k e y_{k}}$ due to the 
unknown information $M S_{k e y_{j}}$. On the other hand, the $\mathcal{A}$ cannot compute $R_{l}$ using $L_{p}$ due to the unknown information $P S_{k_{k e y_{k}}}$. In the same way, the $\mathcal{A}$ cannot find the information $I D_{p}$. Therefore, the $\mathcal{A}$ could not get any confidential information even if he/she has the message $\left\langle L_{p}, N_{p}, O_{p}, I D_{j}, T_{s}, T I D_{p}\right\rangle$.

- Case 4. Similar to Case 3 , the $\mathcal{A}$ attempts to find private information used in the protocol using the public messages $\left\langle P_{p}, Q_{p}, S_{p}, T_{k}\right\rangle$ and $\left\langle M_{2}, M_{3}\right\rangle$, where $P_{p}=$ $h\left(I D_{p}\|S K\| I D_{p k}\left\|T_{k}\right\| h\left(I D_{p} \| P S_{k e y_{k}}\right)\right)$, $Q_{p}=R_{l}^{\prime \prime} \oplus h\left(I D_{p} \quad \| P S_{\text {keyk }}\right), S_{p}=S K \oplus$ $h\left(I D_{p}\left\|R_{l}^{\prime \prime}\right\| T_{k}\right)$ and $M_{2}=T I D_{p}^{\prime} \oplus R_{l}, M_{3}=$ $h\left(I D_{p}\left\|T_{I I D_{p}^{\prime}}\right\| r_{l}\right)$. The objective of the $\mathcal{A}$ is to find the private information $\left\langle I D_{p}, S K, P S_{k e y_{k}}, R_{l}\right\rangle$ from $\left\langle P_{p}, Q_{p}, S_{p}, T_{k}\right\rangle$ and $\left\langle M_{2}, M_{3}\right\rangle$. Since all the public information are protected by the hash function $h(\cdot)$, it is extremely difficult to extract the confidential information $\left\langle I D_{p}, S K, P S_{\text {key }_{k}}, R_{l}\right\rangle$. Therefore, our protocol is secure even if the messages $\left\langle P_{p}, Q_{p}, S_{p}, T_{k}\right\rangle$ and $\left\langle M_{2}, M_{3}\right\rangle$ are known to the $\mathcal{A}$

- Case 5. We suppose that the $\mathcal{A}$ captures the messages $\left\langle Y_{1}, Y_{2}, T_{1}, I D_{m j}\right\rangle$ and $\left\langle Y_{3}, Y_{4}, T_{2}\right\rangle$, where $Y_{1}=I D_{p} \oplus$ $h\left(I D_{m j}^{\prime}\left\|T_{1}\right\| M K_{s h r d}\right)$ and $Y_{2}=h\left(I D_{m j}\left\|I D_{m j}^{\prime}\right\|\right.$ $\left.M K_{\text {shrd }} \| T_{1}\right)$ and $Y_{3}=h\left(I D_{p k}\left\|I D_{p}\right\| T_{1} \|\right.$ $\left.P S_{k e y_{j}}\right), Y_{4}=I D_{p} \oplus h\left(I D_{p k}\left\|P S_{k e y_{j}}\right\| T_{1}\right)$. All the private information $I D_{p}, M K_{\text {shrd }}$, and $P S_{k e y_{j}}$ are protected by the $h(\cdot)$. Therefore, our protocol is secured even if the messages $\left\langle Y_{1}, Y_{2}, T_{1}, I D_{m j}\right\rangle$ and $\left\langle Y_{3}, Y_{4}, T_{2}\right\rangle$ are known to the $\mathcal{A}$.

- Case 6. We suppose that the $\mathcal{A}$ captures the message $\left\langle Y_{5}, Y_{6}, T_{3}\right\rangle$, where $I D_{p}^{\prime}=Y_{4} \oplus h\left(I D_{p k}\left\|P S_{k e y_{j}}\right\| T_{1}\right)$, $Y_{3}^{\prime}=h\left(I D_{p k}\left\|I D_{p}^{\prime}\right\| T_{1} \| P S_{k e y_{j}}\right)$. All the parameters of $\left\langle Y_{5}, Y_{6}, T_{3}\right\rangle$ are protected due to the hardness of $h(\cdot)$, so it is infeasible by the $\mathcal{A}$ to compute all the private information $\left\langle S K, P S_{k e y_{j}}, I D_{p}\right\rangle$.

\section{B. Discussion on Security Attacks of Our Protocol}

In this section, we show that our proposed protocol is secure against all the known security threats. For the Case 1 , we have demonstrated that the adversary could not get any advantage even if obtain all the information stored in the smartcard. Therefore, our protocol is secure against smartcard stolen attack. Moreover, the adversary cannot guess patient's secret information (low-entropy)such as identity, password, and biometric from the smartcard information. Therefore, the protocol is protected against the off-line password guessing attack.

We have also noticed in all the situations described in Case 1 to Case 6 that the adversary is unable to retrieve patient's identity $I D_{p}$. Hence, the anonymity of the patient is strongly achieved in our protocol.

In the patient registration phase, the patient only sends his/her $I D_{p}$ to the medical-registration server $M S_{j}$. No information related to the password $P W_{p}$ is sent to the $M S_{j}$, and further, the server cannot obtain $P W_{p}$ from the known information. Hence, our protocol is free from insider attack.

According to [17], the protocol may suffer from impersonation attack provided the adversary can extract some useful private information from the transmitted public messages. We have described Case 1 to Case 6 that the adversary cannot find the private information from the public messages. Therefore, our protocol is robust against patient impersonation attack, medical-server impersonation attack and physicianserver impersonation attack.

In our protocol, we have used a random number $S K$ as a session key, which is negotiated between the entities involved in the protocol. According to our analysis (see the Section V-A), the parameter $S K$ is strongly protected. Hence, our protocol is free from session key security attack. Since we have utilized the concept of the timestamp in our protocol and each entity check the timestamp, so the adversary cannot launch the replay attack.

In the login phase of our protocol, the smartcard of the patient $U_{p}$ first verifies the patient's biometric template and further verifies the identity and password. Afterwards, the smartcard sends the login message to the $M S_{j}$. Therefore, the unauthorized patient cannot generate a valid login message without. Moreover, an authorized patient cannot also login to the medical-server using wrong login credentials. Therefore, if the patient inputs wrong login credentials, the smartcard will detect and notify the patient. This kind of verification reduces communication and computation cost. Hence, our protocol is efficient. On the other hand, each entity performs some operations after verifying the received message from other entity. Therefore, the mutual authentication between all the entities is also satisfied in our protocol.

\section{Protocol Simulation using AVISPA Software}

In this section, we will present the protocol simulation protocol using AVISPA (see [22] for more information about the tool). We omit the inclusion of the HLPSL code for AVISPA, and only present the simulation findings for both OFMC and Cl-AtSe models (see Fig. 8). The findings show that our protocol is SAFE, which implies that the protocol is secure against active and passive attacks, replay attacks and man-in-the-middle attacks.

\begin{tabular}{|l|l|}
\hline SUMMARY & SUMMARY \\
SAFE & SAFE \\
DETAILS & DETAILS \\
BOUNDED_NUMBER_OF_SESSIONS & BOUNDED_NUMBER_OF_SESSIONS \\
PROTOCOL & TYPED_MODEL \\
/home/avispa/web-interfacecomputation/./ & PROTOCOL \\
tempdir/workfileT6hy8b.if & /home/avispa/web-interfacecomputation/./ \\
GOAL & tempdir/workfileGqYqkK.if \\
as_specified & GOAL \\
BACKEND & As Specified \\
OFMC & BACKEND \\
COMMENTS & CL-AtSe \\
STATISTICS & STATISTICS \\
parseTime: 0.00 s & Analysed : 2488 states \\
searchTime: $0.24 s$ & Reachable : 137 states \\
visitedNodes: 27 nodes & Translation: 0.67 seconds \\
depth: 6 plies & Computation: 0.03 seconds \\
\hline \multicolumn{2}{|c|}{ (a) OFMC Results } \\
\multicolumn{2}{|c|}{ (b) CL-AtSe Results }
\end{tabular}

Fig. 8. Simulation results in OFMC and CL-AtSe backends

\section{Protocol Simulation using Scyther Software}

There are a number of protocol verification and simulation tools, such as ProVerif [10] used to ensure protocol correctness, AVISPA [11] to find potential protocol vulnerabilities, 
and Scyther [12], [13]. The latter is an automated tool, which uses pattern refinement to identify instances of falsification. Some key features of Scyther include:

- support for multi-protocol analysis;

- possibility of unbounded verification with guaranteed termination; and

- analysis of infinite sets of traces in terms of patterns.

Scyther takes as input a protocol description that includes a specification of the intended security properties (also known as security claims) for evaluation. The result window shows a summary of the claims in the protocol and the verification results. Here, one can find whether the protocol is correct or false. The tool has been successfully applied in studies such as those of [15], [16].

Figures 9 and 10 present the outputs from the security analysis of the proposed protocol using Scyther.

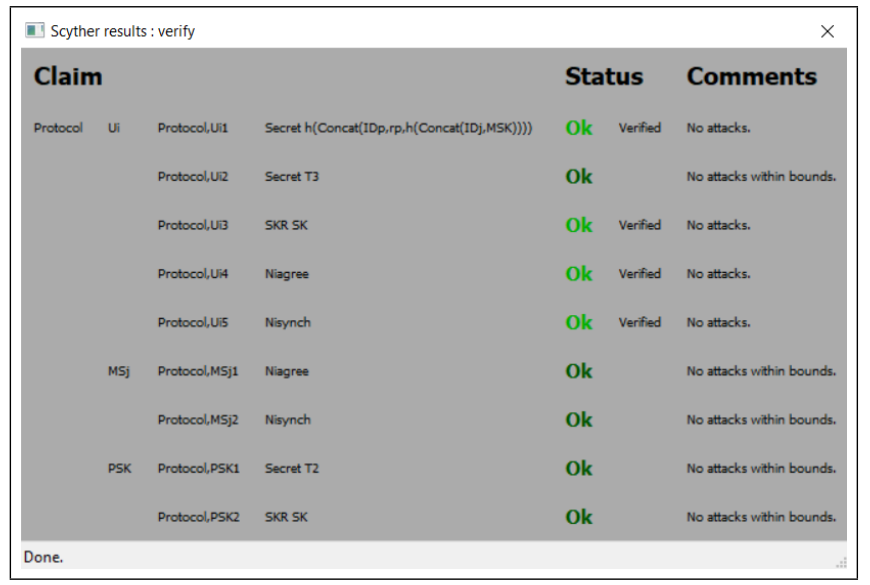

Fig. 9. Security analysis using Scyther for Case 1

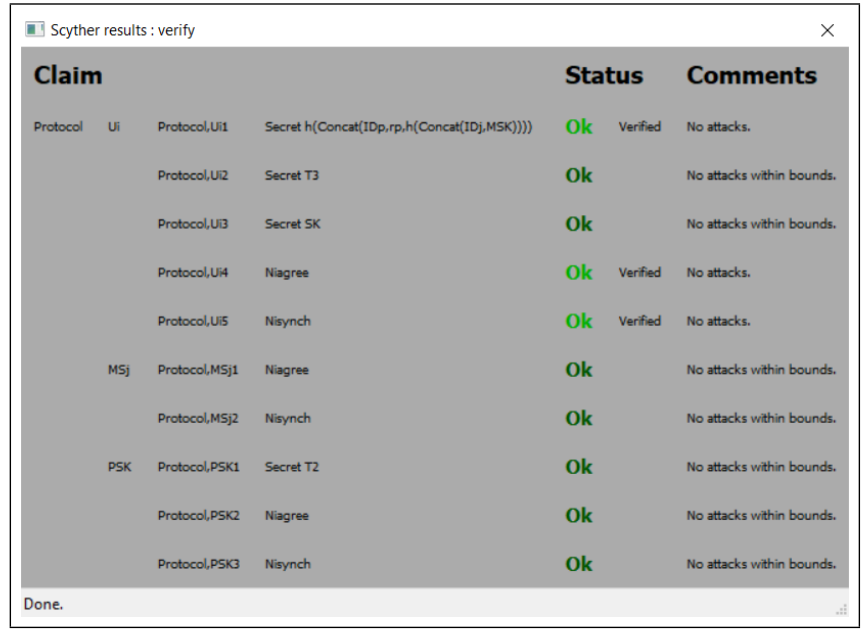

Fig. 10. Security analysis using Scyther for Case 2

\section{Performance Study}

This section discusses the performance of the proposed protocol with related protocols in terms of different security attacks, computation cost, communication cost, and smartcard storage cost. We compare our protocol with Amin and Biswas
[17], Das et al. [18], Maitra et al. [24], Li et al. [25], and Wang and Ma [26] as these protocols use the similar architecture and execute similar operations operations. Several phases are involved in these protocol, but we did not consider the patient registration phase and medical-server registration phase because these phases are executed only one time. In addition to that, the use of password change phase depends on user choice and thus it is also excluded from the comparison.

Note that user anonymity is one of the the most important security attributes, especially to design a secure and robust TMIS system. Besides, protecting user traceability is also equally important. In Das et al.'s protocol [18], they describe that Amin and Biswas [17] protocol is not secure for the several reasons such as insider attack, replay and man-inthe-middle attacks, and design flaws in the registration, login and authentication phases. In this paper, we demonstrate that Das et al.'s protocol does not preserve user anonymity and traceability property. In addition, the same protocol is also vulnerable to smartcard launch attack. Therefore, Das et al.'s protocol is not completely secure, it is not applicable for reallife applications. We then design an authentication protocol and its security analysis claim that the protocol is completely secure including anonymity and traceability under the hardness assumption of the hash function.

TABLE II

COMPUTATION COST COMPARISON

\begin{tabular}{|lllll|}
\hline Protocol & CL & CA & CP & TC \\
\hline Amin and Biswas [17] & $5 T_{h}$ & $14 T_{h}$ & $6 T_{h}$ & 0.0100 \\
Das et al. [18] & $5 T_{h}$ & $15 T_{h}$ & $8 T_{h}$ & 0.0110 \\
Maitra et al. [24] & $4 T_{h}+T_{e}+T_{s}$ & $6 T_{h}+T_{s}$ & $6 T_{h}$ & 2.0900 \\
Li et al. [25] & $2 T_{h}$ & $25 T_{h}$ & $7 T_{h}$ & 0.0130 \\
Wang and Ma [26] & $4 T_{h}+2 T_{s}$ & $7 T_{h}+4 T_{s}$ & $4 T_{h}+2 T_{s}$ & 1.0500 \\
Proposed & $5 T_{h}$ & $\left(16 T_{h} \mid 11 T_{h}\right)$ & $5 T_{h}$ & 0.0084 \\
\hline
\end{tabular}

CL: Computation cost for login phase, CA: Computation cost for authentication phase, CP: Computation cost for password change phase, TC: Total execution cost $(\mathrm{ms})$

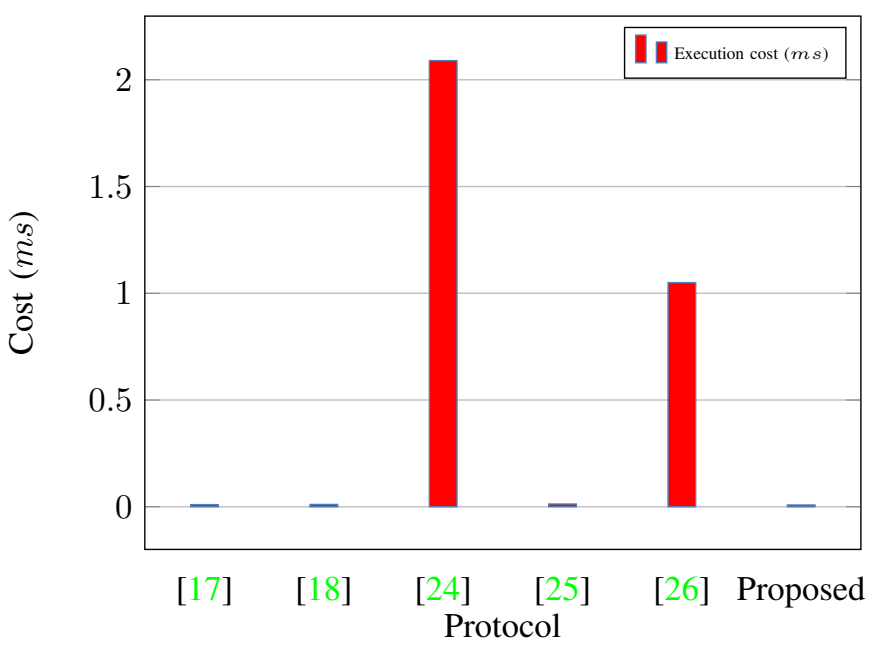

Fig. 11. Total execution cost of different protocols

Now, we evaluate the computation cost of our proposed protocol and existing protocols [17], [18], [24], [25], [26]. Table II and presents the login, authentication, and password change phases. The earlier authentication protocols are vulnerable to 
different attacks. We found from Table II that our protocol takes almost same computation cost in comparison with earlier protocols. Note that our protocol protects all possible security threats by executing the almost same number of hash operation $\left(T_{h}\right)$. It is also noted that our protocol additionally achieves user traceability and anonymity.

We use the experimental results of [23], where the SHA1 hash function has been executed using MIRACL C/C++ Library. The system uses 32-bit Windows 7 OS, Visual C++ 2008 S/W. According to [23], the SHA-1 hash function takes $T_{h} \approx 0.0004$ milliseconds, AES symmetric encryption takes $T_{s} \approx 0.1303$ milliseconds and the exponentiation operation takes $T_{e} \approx 1.8269$ milliseconds. We have also used others Ref. [32], [33], [34] for runtime verification. From Fig. 12 and Table II, it is clear that the protocols in [24], [26] need huge time whereas ours and [18], [25] require very less time.

TABLE III

SMARTCARD STORAGE COST AND COMMUNICATION COST

\begin{tabular}{|llll|}
\hline Protocol & SC (bit) & LC (bit) & AC (bit) \\
\hline Amin and Biswas [17] & $384+(3 \times 128 \times n)$ & 768 & 1152 \\
Das et al. [18] & $768+(3 \times 128 \times n)$ & 480 & 1098 \\
Maitra et al. [24] & $512+(2 \times 128 \times n)$ & 512 & 384 \\
Li et al. [25] & 768 & 512 & 1664 \\
Wang and Ma [26] & 384 & 320 & 256 \\
Proposed & 896 & 640 & $(1536 \mid 1280)$ \\
\hline
\end{tabular}

SC: Smartcard storage cost, LC: Communication cost for login phase, AC: Communication cost for authentication phase, CL: Computation cost for login phase, CA Computation cost for authentication phase, CP: Computation cost for password change phase, $n$ : Total number of medical-servers

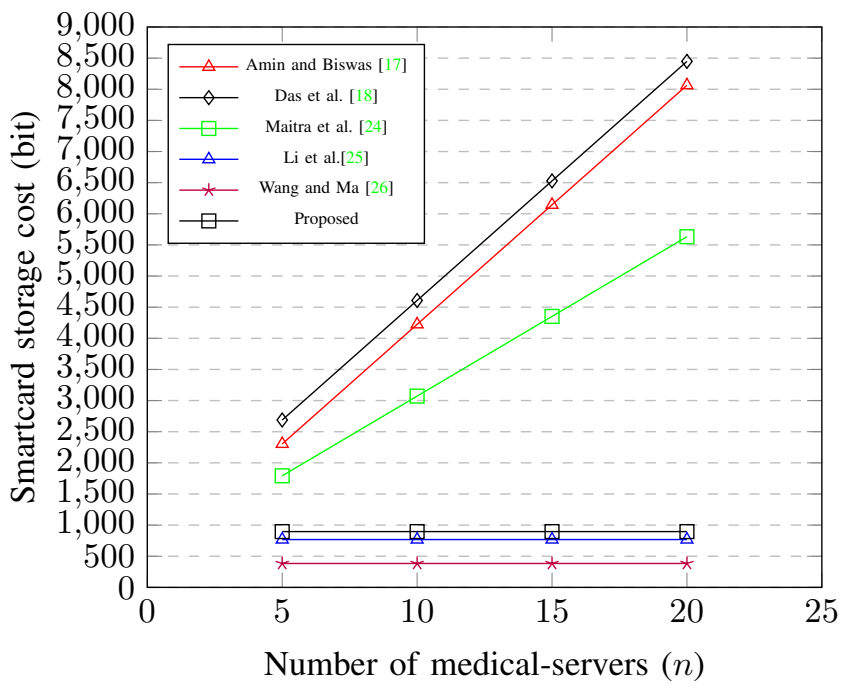

Fig. 12. Smartcard storage cost of different protocols

We evaluate the smartcard storage cost and communication cost of our protocol including previous works in Table III. The storage area in a smartcard is very limited. We found in Table III that the smartcard storage cost in the protocols [17], [18], [24] varies linearly with the number of medical-servers ( $n$ ) whereas our protocol, Li et al.'s protocol [25] and, Wang and Ma [26] are independent of $n$. Because, the architecture of their protocol is different. They have only considered multiple number of servers, whereas the protocols in [17], [18], [24] and

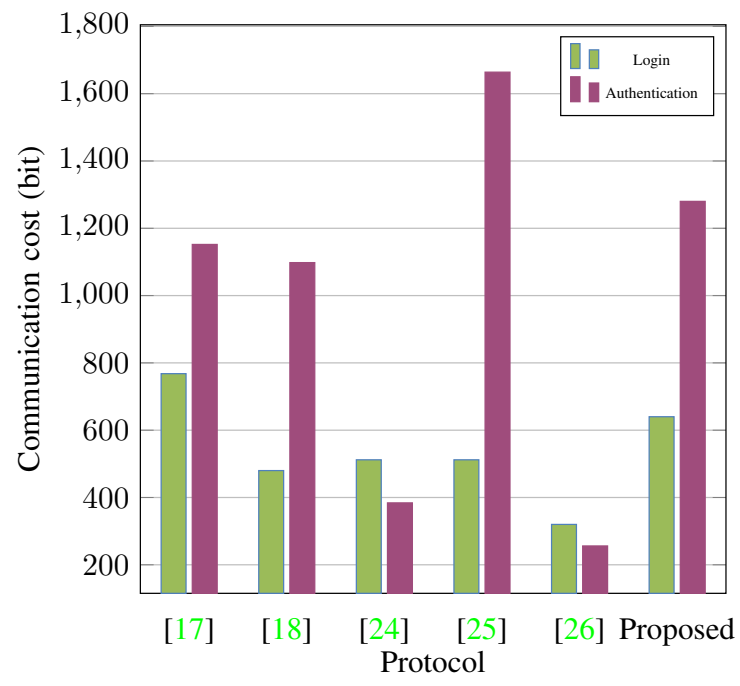

Fig. 13. Communication cost of login and authentication phases of different protocols

ours considered multi-medical systems with multiple number of medical-servers. The major contribution of our protocol is that it is independent of $n$ though it is based on the multiple medical-servers. The pictorial representation of the smartcard storage cost of different protocols including ours are presented in Fig. 12, which shows that our protocol is very efficient compared to the protocols in [17], [18], [24], [25]. In Fig. 13, we also present the communication cost (bit) for login and authentication phases of our protocol and the protocols in [17], [18], [24], [25], [26]. Our protocol is very efficient in terms of communication cost compared to the protocols in [17], [18], [24], [26]. In order to resist the user traceability attack, our protocol incurs additional one communication round. Hence, our protocol takes slightly more communication cost than the existing protocols.

\section{CONCLUSION}

Designing secure cryptographic protocols remains challenging, as demonstrated in this paper (i.e. revealing insecurities in Das et al.'s protocol). More importantly, we proposed an efficient and reliable user authentication protocol for the TMIS environment. Findings from the security and performance evaluations revealed that our protocol is secure against common security threats and provides both user anonymity and user untraceability, as well as being efficient in comparison to related protocols.

Future research includes collaborating with a real-world healthcare organization to implement a prototype of the proposed protocol within a division or department. This will allow us to evaluate its security and performance in a real-world setting, and revise its design if necessary.

\section{REFERENCES}

[1] https://innovatemedtec.com/digital-health/ehealth, last accessed 26 March 2018 
[2] Meng, W., Choo, K.-K. R., Furnell, S., Vasilakos, A. V., \& Probst, C. W. (2018). "Towards Bayesian-based Trust Management for Insider Attacks in Healthcare Software-Defined Networks", IEEE Transactions on Network and Service Management, in press; DOI: 10.1109/TNSM.2018.2815280.

[3] Chuang, M. C., \& Chen, M. C. (2014). "An anonymous multi-server authenticated key agreement scheme based on trust computing using smart cards and biometrics", Expert Systems with Applications, 41(4), 1411-1418.

[4] Li, Xiong, Jianwei Niu, Md Zakirul Alam Bhuiyan, Fan Wu, Marimuthu Karuppiah, and Saru Kumari. "A Robust ECC based Provable Secure Authentication Protocol with Privacy Protection for Industrial Internet of Things." IEEE Transactions on Industrial Informatics (2017).

[5] Shen, Jian, Ziyuan Gui, Sai Ji, Jun Shen, Haowen Tan, and Yi Tang. "Cloud-aided lightweight certificateless authentication protocol with anonymity for wireless body area networks." Journal of Network and Computer Applications (2018).

[6] Rahman, Farzana, Md Zakirul Alam Bhuiyan, and Sheikh Iqbal Ahamed. "A privacy preserving framework for RFID based healthcare systems." Future Generation Computer Systems 72 (2017): 339-352.

[7] He, D., Kumar, N., Chen, J., Lee, C. C., Chilamkurti, N., \& Yeo, S. S. (2015). "Robust anonymous authentication protocol for healthcare applications using wireless medical sensor networks", Multimedia Systems, 21(1), 49-60. Chicago

[8] Mishra, D., Das, A. K., \& Mukhopadhyay, S. (2014). "A secure user anonymity-preserving biometric-based multi-server authenticated key agreement scheme using smart cards. Expert Systems with Applications", 41(18), 8129-8143.

[9] Gope, P., \& Hwang, T. (2016). "A realistic lightweight anonymous authentication protocol for securing real-time application data access in wireless sensor networks", IEEE Transactions on Industrial Electronics, 63(11), 7124-7132.

[10] Blanchet, Bruno. "An Efficient Cryptographic Protocol Verifier Based on Prolog Rules", csfw. Vol. 1. 2001.

[11] Armando, Alessandro, et al. "The AVISPA tool for the automated validation of internet security protocols and applications", International conference on computer aided verification. Springer, Berlin, Heidelberg, 2005.

[12] Cremers, Cas JF. "The scyther tool: Verification, falsification, and analysis of security protocols", CAV. Vol. 8. 2008

[13] Cremers, Cas JF. "Scyther: Semantics and verification of security protocols. Eindhoven", Netherlands: Eindhoven University of Technology, 2006.

[14] Gope, Prosanta, and Ruhul Amin. "A novel reference security model with the situation based access policy for accessing ephr data." Journal of medical systems 40, no. 11 (2016): 242.

[15] Ali, Mazhar, Saif Malik, and Samee Khan. "DaSCE: Data security for cloud environment with semi-trusted third party", IEEE Transactions on Cloud Computing (2015).

[16] Cao, Jin, Maode Ma, and Hui Li. "G2RHA: Group-to-Route Handover Authentication Scheme for Mobile Relays in LTE-A High-Speed Rail Networks", IEEE Transactions on Vehicular Technology 66.11 (2017): 9689-9701.

[17] Amin, R., \& Biswas, G. P. (2015). "A novel user authentication and key agreement protocol for accessing multi-medical server usable in tmis", Journal of medical systems, 39(3), 33.

[18] Das, A. K., Odelu, V., \& Goswami, A. (2015). "A secure and robust user authenticated key agreement scheme for hierarchical multi-medical server environment in TMIS", Journal of medical systems, 39(9), 92.

[19] Lin, Hao, Fengtong Wen, and Chunxia Du. "An Improved User Authentication and Key Agreement Scheme for Multi-medical Server Usable in TMIS.” DEStech Transactions on Engineering and Technology Research iect (2016).

[20] Liu, X., Li, Y., Qu, J. and Ding, Y., 2017. A Lightweight Pseudonym Authentication and Key Agreement Protocol for Multi-medical Server Architecture in TMIS. KSII Transactions on Internet \& Information Systems, 11(2).

[21] Amin, R., \& Biswas, G. P. (2016). "A secure light weight scheme for user authentication and key agreement in multi-gateway based wireless sensor networks", Ad Hoc Networks, 36, 58-80.

[22] Armando, A. et al., "The AVISPA tool for the automated validation of internet security protocols and applications", In: Proceedings of the 17th International conference on computer aided verification, LNCS, vol. 3576, pp. 281285, 2005.

[23] Xu, L., Wu, F., "Cryptanalysis and Improvement of a User Authentication Scheme Preserving Uniqueness and Anonymity for Con- nected Health Care", Journal of Medical Systems, 39: 10, 2015. DOI 10.1007/s10916-014-0179-x

[24] Maitra, T., and Giri, D., "An efficient biometric and passwordbased remote user authentication using smart card for telecare medical information systems in multi-server environment”. J. Med. Syst. 38(12):142, 2014. doi:10.1007/s10916-014-0142-x.

[25] X.Li, Y.P.Xiong, J.Ma, W.D.Wang, "An efficient and security dynamic identity based authentication protocol for multi-server architecture using smartcards", Journal of Network and Computer Applications 35(2) (2012)763-769.

[26] B.Wang, and M. Ma, "A Smart Card Based Efficient and Secured MultiServer Authentication Scheme", Wireless Personal Communications, vol. 68, no. 2, pp. 361-378, 2013.

[27] S.H. Islam, A.K. Das, M.K. Khan. "An efficient biometric-based password authentication scheme for client-server environment using ECC and fuzzy extractor", International Journal of Ad Hoc and Ubiquitous Computing, 2015. DOI: 10.1504/IJAHUC.2015.10001794

[28] S.H. Islam, A.K. Das, M.K. Khan. "Design of a provably secure identity-based digital multi-signature scheme using biometrics and fuzzy extractor", Security and Communication Networks, 9(16), 3229-3238, 2016.

[29] X. Li, J-W. Niu, J. Ma, W-D. Wang, C-L. Liu. Cryptanalysis and improvement of a biometrics-based remote user authentication scheme using smart cards. Journal of Network and Computer Applications, 34(1):73-79, 2011.

[30] P. Kocher, J. Jaffe, and B. Jun, Differential power analysis, Proceedings of the Advances in Cryptology(CRYPTO'99), LNCS, vol. 1666, pp. 388-397, 1999.

[31] T.S. Messerges, E.A. Dabbish, R.H. Sloan, Examining smartcard security under the threat of power analysis attacks. IEEE Transactions on Computers, 51(5):541-552, 2002.

[32] Jiang, Yu, Houbing Song, Rui Wang, Ming Gu, Jiaguang Sun, and Lui Sha. "Data-centered runtime verification of wireless medical cyberphysical system." IEEE transactions on industrial informatics 13 , no. 4 (2017): 1900-1909.

[33] Ou, Andrew Y-Z., Maryam Rahmaniheris, Yu Jiang, Po-Liang Wu, and Lui Sha. "Toward safe interoperations in network connected medical cyber-physical systems using open-loop safe protocols." In Proceedings of the 36th International Conference on Computer-Aided Design, pp. 957-963. IEEE Press, 2017.

[34] Jiang, Yu, Mingzhe Wang, Han Liu, Mohammad Hosseini, and Jiaguang Sun. "Dependable integrated clinical system architecture with runtime verification." In Computer-Aided Design (ICCAD), 2017 IEEE/ACM International Conference on, pp. 951-956. IEEE, 2017.

[35] Chunyong Yin, Jinwen Xi, Ruxia Sun, Jin Wang. "Location Privacy Protection Based on Differential Privacy Strategy for Big Data in Industrial Internet of Things ." IEEE Transactions on Industrial Informatics, 14(8), pp. 3628-3636, 2017.

[36] Ping Guo, Jin Wang, Sai Ji, Xue Hua Geng, Neal N. Xiong. ”A Lightweight Encryption Scheme Combined with Trust Management for Privacy-Preserving in Body Sensor Networks". Journal of Medical Systems, 39(12), pp.190-198, 2015.

[37] Jian Shen, Haowen Tan, Sangman Moh, Ilyong Chung, Jin Wang. "An Efficient RFID Authentication Protocol Providing Strong Privacy and Security”, Journal of Internet Technology, 17(3), 2016

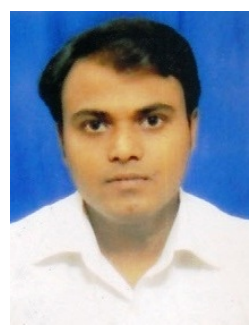

Ruhul Amin received Ph.D. in Computer Science and Engineering from the Indian Institute of Technology [IIT(ISM)] Dhanbad, Jharkhand, India, in 2017. He also received B.Tech. and M.Tech. both in Computer Science and Engineering from Maulana Abul Kalam Azad University of Technology, West Bengal, India in 2009 and 2013, respectively. Presently, he is working as an Assistant Professor in the Department of Computer Science and Engineering, Dr. Shyama Prasad Mukherjee International Institute of Information Technology, Naya Raipur, India. His research interest includes authentication protocol and security in WSNs. 


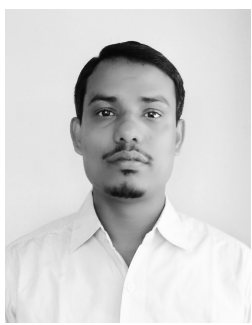

SK Hafizul Islam (SM'18) received the M.Sc. degree in Applied Mathematics from Vidyasagar University, Midnapore, India, in 2006, and the M.Tech. degree in Computer Application and the Ph.D. degree in Computer Science and Engineering in 2009 and 2013, respectively, from the Indian Institute of Technology [IIT (ISM)] Dhanbad, Jharkhand, India, under the INSPIRE Fellowship Ph.D. Program (funded by Department of Science and Technology, Government of India). He is currently an Assistant Professor with the Department of Computer Science and Engineering, Indian Institute of Information Technology Kalyani (IIIT Kalyani), West Bengal, India. Before joining the IIIT Kalyani, he was an Assistant Professor with the Department of Computer Science and Information Systems, Birla Institute of Technology and Science, Pilani (BITS Pilani), Rajastahn, India. He has more than five years of teaching and eight years of research experiences. He has authored or co-authored seventy five research papers in journals and conference proceedings of international reputes. His research interests include cryptography, information security, WSNs, IoT, and cloud computing. Dr. Islam is an Associate Editor for Wiley's International Journal of Communication Systems and Security and Privacy. He was a reviewer in many reputed international journals and conferences. He was the recipient of the University Gold Medal, the S.D. Singha Memorial Endowment Gold Medal, and the Sabitri Parya Memorial Endowment Gold Medal from Vidyasagar University, in 2006. He was also the recipient of the University Gold Medal from IIT(ISM) Dhanbad in 2009 and the OPERA award from BITS Pilani in 2015. He is a member of IEEE and ACM.

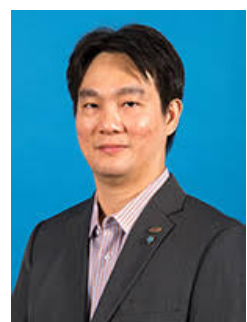

Kim-Kwang Raymond Choo received the Ph.D. in Information Security in 2006 from Queensland University of Technology, Australia. He currently holds the Cloud Technology Endowed Professorship at The University of Texas at San Antonio, and an adjunct associate professorship at the University of South Australia. He serves on the editorial board of Computers \& Electrical Engineering, Cluster Computing, Digital Investigation, IEEE Access, IEEE Cloud Computing, IEEE Communications Magazine, Future Generation Computer Systems, Journal of Network and Computer Applications, PLoS ONE, Soft Computing, etc. $\mathrm{He}$ also serves as the Special Issue Guest Editor of ACM Transactions on Embedded Computing Systems (2017), ACM Transactions on Internet Technology (2016), Computers and Electrical Engineering (2017), Digital Investigation (2016), Future Generation Computer Systems (2016,2018), IEEE Cloud Computing (2015), IEEE Network (2016), IEEE Transactions on Cloud Computing (2017), IEEE Transactions on Dependable and Secure Computing (2017), Journal of Computer and System Sciences (2017), Multimedia Tools and Applications (2017), Personal and Ubiquitous Computing (2017), Pervasive and Mobile Computing (2016), Wireless Personal Communications (2017), etc. In 2016, he was named the Cybersecurity Educator of the Year APAC (Cybersecurity Excellence Awards are produced in cooperation with the Information Security Community on LinkedIn), and in 2015 he and his team won the Digital Forensics Research Challenge organized by Germany's University of Erlangen-Nuremberg. He is the recipient of ESORICS 2015 Best Paper Award, 2014 Highly Commended Award by the Australia New Zealand Policing Advisory Agency, Fulbright Scholarship in 2009, 2008 Australia Day Achievement Medallion, and British Computer Society's Wilkes Award in 2008. He is also a Fellow of the Australian Computer Society, and a Senior Member of IEEE.

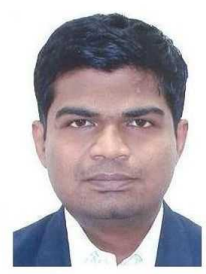

Nachiket Tapas received the M.Tech. degree in computer science and engineering from the National Institute of Technology Goa, India, in 2016. He is currently persuing Ph.D. from the Department of Engineering, University of Messina, Italy 98158 Prior to this, Mr. Tapas served more than four year as a Senior Software Engineer at Infosys Technologies Limited, Bangalore. His research interests include Internet of Things, Data Mining, and security in mobile communication and cloud computing. 